\title{
A New Energy-Efficient Coverage Control with Multinodes Redundancy Verification in Wireless Sensor Networks
}

\author{
Zeyu Sun, ${ }^{1,2}$ Chuanfeng Li, ${ }^{1,3}$ Xiaofei Xing, ${ }^{4}$ and Huihui Wang 5 \\ ${ }^{1}$ School of Computer and Information Engineering, Luoyang Institute of Science and Technology, Henan 471023, China \\ ${ }^{2}$ School of Electronics and Information Engineering, Xian Jiaotong University, Shaanxi 710049, China \\ ${ }^{3}$ School of Electronics, Electrical Engineering and Computer Science, Queen's University Belfast, Belfast BT95AH, UK \\ ${ }^{4}$ School of Computer Science and Educational Software, Guangzhou University, Guangdong 510006, China \\ ${ }^{5}$ Department of Engineering, Jacksonville University, 2800 University Blvd N, Jacksonville, FL 32211, USA
}

Correspondence should be addressed to Huihui Wang; hwang1@ju.edu

Received 5 September 2016; Accepted 27 October 2016

Academic Editor: Jesus Corres

Copyright (c) 2016 Zeyu Sun et al. This is an open access article distributed under the Creative Commons Attribution License, which permits unrestricted use, distribution, and reproduction in any medium, provided the original work is properly cited.

The target nodes are $k$-covered by the sensor nodes; there will be a lot of redundant data forcing the phenomenon of congestion which lowers the network communication capability and coverage and accelerates network energy consumption. Therefore, this paper proposes an energy-efficient coverage control with multinodes redundancy verification (ECMRV). The algorithm constructs the coverage network model by using the positional relationship among the sensor nodes, and the proof procedure of the sector coverage expected value of the monitoring area also has been provided. On the aspect of the energy consumption, this paper gives the proportion of energy conversion functions between the working sensor nodes and the neighboring sensor nodes. And by using the function proportional to schedule the sensor nodes with low energy, we achieve the energy balance of the whole network and the optimization of the network resources. Finally, the simulation experiments indicate that this algorithm can not only improve the quality of network coverage, but completely inhibit the rapid energy consumption of the sensor nodes as well; as a result, the network lifetime extends, which has verified availability and stability of the algorithm in the paper.

\section{Introduction}

Wireless Sensor Network is a new type of network architecture, which is formed by thousands of sensor nodes [1-3]. Each sensor node has the abilities of sensing, computing, communicating, and storing and the behavioral characteristic is to complete the communication transmission by the multihop method in the information world, which makes data acquisition, data storage, data computing, and communication transmission link network service system come into being $[4,5]$.

The coverage quality and energy consumption are two important indices of evaluating the performance of Wireless Sensor Networks system. Coverage quality can be directly reflected in the deployment of the sensor nodes in the monitoring area. And the network lifetime directly affects the quality of the entire network service, which is mainly reflected in the energy consumption of the sensor nodes deployment. Generally, because of the restriction of the topography, environmental factors, and many other constraints, the selection of the sensor node deployment is random. In the random deployment process, due to the unpredictable sensor node location information in advance, it is possible to make a monitoring target node multiply covered, namely, $k$-coverage. Take the military battlefield as example, as shown in Figure 1. And there is another situation, due to the randomness; it is possible to make a monitoring area a blind one. The only way to achieve complete coverage is to add more sensor nodes. Although the two ways above can achieve complete coverage of the target to a certain extent, there are still some shortcomings. First, because of the existence of the $k$-coverage, in the process of data collection and calculation and in the data transmission of the communication linkage, there will be a large amount of redundant data, which cause larger error and uncertainty. Second, the real meaning of the coverage is not the complete 


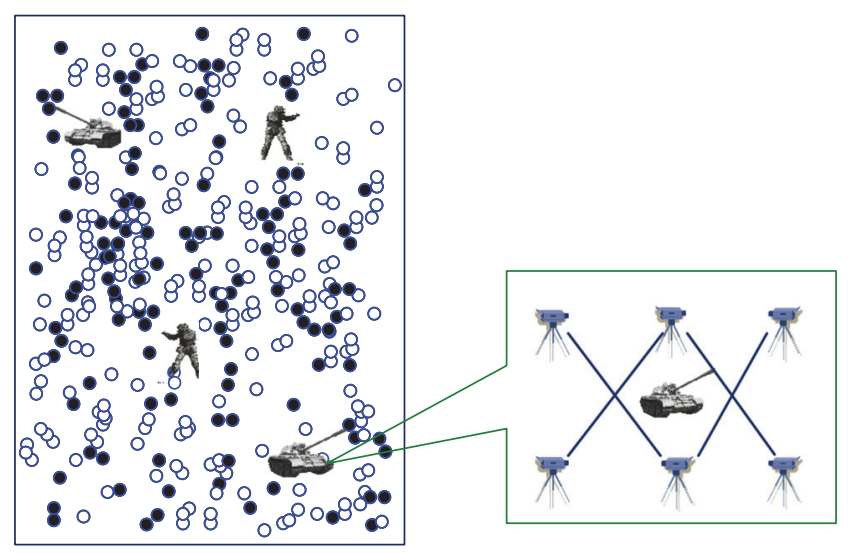

FIGURE 1: The topological structure of the coverage in the military battlefield.

coverage of the entire monitoring area, but the complete coverage of the certain target nodes; in the process of covering the entire monitoring area completely, to take no account of the existence of the target nodes will consume a lot of sensor node energy, which will accelerate the speed of the collapse of the whole network system. Third, when deploying the sensor nodes, the existence of the randomness will inevitably cause excessive density in a certain coverage area of the monitoring area, resulting in the bottlenecking, which eventually generates information redundancy verification in the channel but reduces the network expansibility.

Aiming at the above shortages, this paper proposed the energy-efficient coverage control with multinodes redundancy verification (ECMRV). In this paper, the coverage probability and covering expected value of sensor nodes are solved by using the sector domains of the coverage area when the moving target nodes are passing through the monitoring area; as for the energy consumption, this paper gives the solution of multipoint transmission and single point transmission. For the working sensor nodes, having met the certain coverage ratio, when the moving target nodes are passing across the $k$-coverage area, it will close the sensor nodes within or higher the threshold value or it will place the sensor nodes in the sleeping state by the sensor node adaptive transformation mechanism and the other sensor nodes will use the sensor node energy scheduling mechanism to compete the conversion of the energy of the sensor nodes, so that the network lifetime can be extended.

\section{Related Knowledge}

The technique of coverage control in WSNs is a very important fundamental research and also one of the hotspot problems right now in WSN. The coverage quality will affect the lifetime of the network. In recent years, many experts and scholars at home and broad have carried out plenty of researches thoroughly and carefully. Literature [6] brings in the partial coverage optimization and constitutes the partial $\alpha$ coverage, and after a series of calculation and optimization of the $\alpha$, the complete coverage optimization is finally achieved. All the above-mentioned algorithms have achieved the effective coverage of the monitoring area to some extent; however they have the mutual problem that the algorithms have more calculations, and they are more complex, and the scalability of the Wireless Sensor Network is worse. Literature [7] provides a constructive method of connected coverage protocol. The protocol provides the proportional relation between the network coverage quality and the network and the performance indexes of all the parameters in the network system and creates the scheduling control algorithm (SCA), the purpose of which is to use the least sensor nodes to guarantee the connection of the whole network system. Literature [8] introduces the artificial intelligence algorithms, that is, the swarm intelligence algorithm and the granule algorithm to complete the sensor nodes deployment of the whole network monitoring area and in the stage of the coverage optimization, the whole coverage is optimized by the two artificial intelligence algorithms and finally realizes the complete coverage of the monitoring area; as for the energy consumption, it completes the scheduling conversion of the sensor nodes energy by the heuristic sensor nodes Scheduling, which extends the lifetime of the network. Literature [9] proposes the optimization strategy on coverage algorithm based on Voronoi. Satisfying the condition of a certain coverage quality, the algorithm adds some work sensor nodes to the coverage hole so as to improve the current coverage ratio; meanwhile, it will search reasonable information of repairing site to guarantee the connection of the whole network. Literature [10] also takes the Voronoi as the research object and solves the information of wire rod site that formed by the geometry variation in the Voronoi and the sensor nodes and finally completes the coverage of the monitoring area. Literature [11] and literature [12] compute the goal sensor nodes effectively, utilizing the different angles of the sector composed by the sensor nodes and the target nodes, and give the method of computing the coverage probability of different monitoring area. All algorithms mentioned above have the better feasibility, the higher reliability, and the larger network scalability. However, in the research process of the four algorithms, their network models are much too idealized. From literature [9] to literature [11], they all take the static target nodes as the research objective, and literature [12] does not consider the situation when the moving target nodes are the concerned target nodes, which result in the $k$ barrier coverage. Literature [13] proposes the polytypic target coverage algorithm based on the linear programming. The algorithm utilizes the clustering structure to solve the polytypic target coverage problem. By computing the coverage ability and the residual energy of the sensor, it completes the polytypic target optimization coverage in the way of the linear programming. Literature [14] proposes the event probability driven mechanism algorithm. This algorithm calculated the coverage probability and the expected value of the sensor nodes with the probability model, then optimized the result, and finally achieved the optimal coverage. The above two algorithms achieve the purposes of the optimal coverage and the extension of the network lifetime, but the coverage condition is a little hasher, and the calculations of the algorithms are much more complex. 
In order to make the effective coverage of the monitoring area better, on the basis of literature [11] and literature [12], this paper proposes the energy-efficient coverage control with multinodes redundancy verification (ECMRV). Taking use of sectorial the $k$-degree coverage, the algorithm calculates the coverage expected value between the sensor nodes and the moving target nodes. And then, the algorithm provides the solving procedure of the coverage expected value when a certain sensor node finishes the coverage of the target nodes. In terms of the energy, on the basis of the analysis of all the sensor nodes' energy in the whole network, the algorithm provides the process of the energy comparison under the multilateral and unilateral connection; that is, the energy consumption of the data in multicast is less than that of the unilateral sensor nodes. In terms of the energy conversion, the algorithm completes the energy conversion among the sensor nodes through the self-scheduling mechanism, extending the lifetime of the network. Finally, through the simulation experiments and the comparison with other algorithms, the effectiveness and the stability of the ECMRV algorithm have been verified.

\section{The Network Model and the Coverage Quality}

3.1. Basic Concepts and Basic Theories. For the sake of the better explanation and research of the WSNs coverage problems and the convenience of the problems, the ECMRV algorithm is based on the following four hypothesizes $[10,15,16]$ :

(1) All of the sensor nodes are all in the homogeneous form, and the perceived range and the communication range are both disc-shaped.

(2) All of the sensor nodes can get their own position information through the GPS technology.

(3) The initial energy of all the sensor nodes is the same and has the same sensing range, and the speed is the same as that of the clock.

(4) The sensor nodes are deployed randomly in the square monitoring area with the length $l$, and the perceived range must be guaranteed to be less than the length $l$, and the boundary effect can be ignored.

(5) The sensor nodes are randomly deployed in the monitoring areas with high density, and the sensor nodes are independent of each other.

Definition 1 (the complete coverage). In the monitoring area, any target nodes must be covered by at least one sensor node; that is, the Euclidean distance of the sensor nodes and the target nodes are smaller than the perceived range of the sensor nodes, $d(i, t) \leq R_{i}$, which is called complete coverage.

Definition 2 (the $k$-coverage). In the monitoring area, any target nodes must be covered by at least $k$ sensor nodes. Thus, the monitoring area is called $k$-degree coverage area.

Definition 3 (the coverage quality). In the monitoring area, the coverage quality is the specific value of the sum of the sensed area of all the sensor nodes and the area of the monitoring area. In a way, it reflects the coverage degree of the target nodes.

Definition 4 (the multilevel coverage). The coverage rate of each sensor node is $p$; then the coverage rate at any point in a two-dimensional plane is

$$
p_{n}=1-(1-p)^{n} \text {. }
$$

In (1), $p_{n}$ is the multilevel coverage ratio; $p$ is the coverage probability of any one of the sensor nodes; $n$ is the number of the sensor nodes $[10,17,18]$.

Theorem 5. When the moving target nodes enter the monitoring area for the first time, the first coverage expected value is $E(X)=\left[1-(1-p)^{N}\right] p^{-1}$, and $N$ is the biggest number of times that the moving target nodes can complete the transmission, and $p$ is the coverage probability of the sensor nodes.

Proof. According to the probability theory, suppose that $X$ represents how many times the moving target nodes move and the value range of $X$ is possible to be $X \in[1,2,3, \ldots, N]$. When $X=m$ as well as meeting the condition of $1 \leq m \leq$ $N-1$, that is, when the first $N-1$ moving target nodes are not covered by the sensor nodes, the distribution density function of $X$ will be

$$
P(X=k)= \begin{cases}p(1-p)^{k-1}, & k=1,2,3, \ldots, N-1 \\ (1-p)^{N-1}, & k=N .\end{cases}
$$

According to the equation of the coverage, thus

$$
E(X)=\sum_{k=1}^{N-1} k p(1-p)^{k-1}+N(1-p)^{N-1} .
$$

Make $q=1-p$ and $S=\sum_{k=1}^{N-1} k(1-p)^{k-1}$; that is, $S=$ $\sum_{k=1}^{N-1} k q^{k-1}$ is multiplied by $q$ in both sides of the equation; thus $q S=\sum_{k=1}^{N-1} k q^{k}$; that is,

$$
\begin{aligned}
S & =\frac{1-q^{N-1}}{(1-q)^{2}}-\frac{(N-1) q^{N-1}}{1-q} \\
& =\frac{1-(1-p)^{N-1}}{p^{2}}-\frac{(N-1)(1-p)^{N-1}}{p} .
\end{aligned}
$$

Put (4) into (3); thus,

$$
\begin{aligned}
E(X)= & p\left(\frac{1-(1-p)^{N-1}}{p^{2}}-\frac{(N-1)(1-p)^{N-1}}{p}\right) \\
& +N(1-p)^{N-1}=\left[1-(1-p)^{N}\right] p^{-1} .
\end{aligned}
$$

In the square monitoring area, after a period of $t$, the energy of the working sensor nodes must have been consumed by themselves, which make the coverage area change correspond. In order to suppress the energy consumption of the sensor nodes and extend the lifetime of the network as 


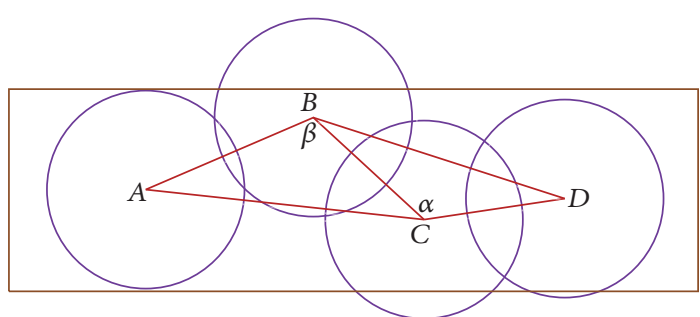

FIgURE 2: Multilateral and unilateral node connection.

long as possible, this paper adopts the sensor node energy model to analyze the sensor node-energy that is consumed in the process of unilateral and multilateral data transmission; meanwhile, the energy of the sensor node is consumed by its own work of calculation [19-22], storage, and control. The energy consumption model of the sensor node transmitter is

$$
\begin{aligned}
E_{\mathrm{Tx}}(l, d) & =l E_{T-\text { elec }}+E_{\text {amp }}(l, d) \\
& = \begin{cases}l E_{T-\text { elec }}+l \varepsilon_{f s} d^{2}, & d<d_{0} \\
l E_{T-\text { elec }}+l \varepsilon_{\text {amp }} d^{4}, & d \geq d_{0} .\end{cases}
\end{aligned}
$$

And the energy consumption model of the receiver is

$$
E_{\mathrm{Rx}}(l)=E_{R \text {-elec }}(l)=l E_{\text {elec }} .
$$

And $l$ is the bit of the data transmission, $d$ is the Euclidean distance between the sensor nodes and the neighboring nodes, and $d_{0}$ is the threshold value of the distance of the communication sensor node. When the distance of the communication sensor node is smaller than $d_{0}$, the energy damping index is 2, whereas the energy damping index will be $4 . E_{\text {elec }}$ represents the energy consumption of communication sensor nodes receiving and transmitting modules $[23,24]$.

Theorem 6. Less energy of the data is consumed in multicast than the unilateral sensor nodes. The mathematical induction when $n \rightarrow \infty$ is shown in Figure 2.

In 1st case, according to formula (6), the formula of energy dissipations directly from source node $A$ to target node $D$ is presented as below:

$$
E_{\mathrm{Tx} A D}\left(l, d_{A D}\right)= \begin{cases}l E_{T-\text { elec }}+l \varepsilon_{f s} d_{A D}^{2}, & d_{A D}<d_{0} \\ l E_{T \text {-elec }}+l \varepsilon_{\mathrm{amp}} d_{A D}^{4}, & d_{A D} \geq d_{0} .\end{cases}
$$

In the 2nd case, data are sent from node $A$ to $B$ and then to $D$. From $A$ to $B$, when $k=3$, the model of energy consumption when transmitting module in node $A$ can be inducted as follows:

$$
\begin{gathered}
E_{\mathrm{Tx} \_A B}\left(l, d_{A B}\right)= \begin{cases}l E_{T-\text { elec }}+l \varepsilon_{f_{s}} d_{A B}^{2}, & d_{A B}<d_{0} \\
l E_{T-\text { elec }}+l \varepsilon_{\mathrm{amp}} d_{A B}^{4}, & d_{A B} \geq d_{0}\end{cases} \\
E_{\mathrm{Rx} B B}(l)=l E_{\text {elec }} .
\end{gathered}
$$

The energy consumption of node $B$ receiving the information is represented as below:

$$
E_{\mathrm{Tx} \_B D}\left(l, d_{B D}\right)= \begin{cases}l E_{T-\text { elec }}+l \varepsilon_{f s} d_{B D}^{2}, & d_{B D}<d_{0} \\ l E_{T-\text { elec }}+l \varepsilon_{\mathrm{amp}} d_{B D}^{4}, & d_{B D} \geq d_{0} .\end{cases}
$$

Then, the total energy consumption from node $A$ to $B$ and then to $D$ is represented as below:

$$
E_{\mathrm{Total}}=E_{\mathrm{Tx} \_A B}+E_{\mathrm{Rx} \_B}+E_{\mathrm{Tx} \_B C} .
$$

In their initial state, the nodes work with the same amount of energy and are independent of each other. The basic properties of triangle are shown.

At the beginning, each node is independent of each other and is working with equal energy. This basic feature of forming triangles can be represented as below:

$$
E_{\mathrm{Total}}=E_{\mathrm{Tx} \_A B}+E_{\mathrm{Rx} \__{-} B}+E_{\mathrm{Tx} \__{-} B D}<E_{\mathrm{Tx} \__{-} A D} .
$$

That is to say, when $k=3$, less energy is consumed in multilateral data transmission than in unilateral one. So when $k=3$, formula (12) is justified.

The data transmitting path is from $A$ to $B$ to $C$ and then to $D$. When $n \rightarrow \infty$, because $\angle \alpha$ is an obtuse angle, $\angle \alpha>$ $\pi / 2, \cos \angle \alpha<0$. According to Cosine Theorem, $B C^{2}+C D^{2}<$ $B D^{2}$. The triangle of connecting $B, C$, and $D$ is obtuse triangle. Hence, formula (13) is obtained as below:

$$
\begin{gathered}
E_{\mathrm{Tx}_{-} A B}+E_{\mathrm{Rx}_{-} B}+\cdots+E_{\mathrm{Rx}_{n}-1}+E_{\mathrm{Tx}_{n}-1, n}<\cdots \\
\quad<E_{\mathrm{Tx}_{A B}}+E_{\mathrm{Rx}_{B}}+E_{\mathrm{Tx}_{B D}}<E_{\mathrm{Tx}_{A D}} .
\end{gathered}
$$

\subsection{Cover Quality}

Theorem 7. Randomly select $k$ sensor nodes within $M$, and then the expectation of the coverage quality is

$$
E\left(\eta_{k}\right)=1-\left\{1-\frac{\pi\left(R_{0}^{2}+\lambda^{2}\right)}{\operatorname{area}(M)}\right\}^{k} .
$$

Proof. The sensor node subject to the random uniform distribution in $M$, so the probability a sensor node is located in any position in $M$, is $1 / \operatorname{area}(M)$. If any point $q$ is covered by a sensor node $a$ (the perception radius is denoted with $R_{a}$ ), then sensor node $a$ must be locate in a circle of centered on $q$ (the circle is denoted as $O\left(q, R_{a}\right)$ of radius $\left.R_{a}\right)$. Therefore, the probability that $q$ is covered by sensor node $a$ is

$$
P_{a}=\frac{\operatorname{area}\left(O\left(q, R_{a}\right)\right)}{\operatorname{area}(M)} .
$$

Because the perception radius of sensor node $a$ is normal distribution and $R_{0} \geq 3.3 \lambda$, the probability that any point is covered by a work sensor node is

$$
P=\int_{0}^{2 R_{0}} P_{a} \frac{1}{(2 \pi)^{1 / 2} \lambda} \exp \left(-\frac{\left(R_{a}-R_{0}\right)}{2 \lambda^{2}}\right) \mathrm{d} R_{0} .
$$


Let $x=\left(R_{a}-R_{0}\right) / \lambda$; then

$$
\begin{aligned}
P & =\left(\frac{\pi}{2}\right)^{1 / 2} \frac{1}{\operatorname{area}(M)}\left(\int_{-R_{0} / \lambda}^{R_{0} / \lambda} \lambda^{2} x^{2} \exp \left(-\frac{x^{2}}{2}\right) \mathrm{d} x\right. \\
& +\int_{-R_{0} / \lambda}^{R_{0} / \lambda} 2 \lambda x R_{0} \exp \left(-\frac{x^{2}}{2}\right) \mathrm{d} x \\
& \left.+\int_{-R_{0} / \lambda}^{R_{0} / \lambda} R_{0}^{2} \exp \left(-\frac{x^{2}}{2}\right) \mathrm{d} x\right) .
\end{aligned}
$$

Calculation is as follows:

$$
\begin{aligned}
P & =\left(\frac{\pi}{2}\right)^{1 / 2} \frac{1}{\operatorname{area}(M)}\left(-\left.\lambda^{2} \exp \left(-\frac{x^{2}}{2}\right)\right|_{-R_{0} / \lambda} ^{R_{0} / \lambda}\right. \\
& \left.+(2 \pi)^{1 / 2} \lambda+(2 \pi)^{1 / 2} R_{0}^{2}\right) \approx \frac{\pi\left(R_{0}^{2}+\lambda^{2}\right)}{\operatorname{area}(M)} .
\end{aligned}
$$

The positions of all sensor nodes in $M$ are independent of each other, according to formula (1), the probability that any sensor node in $M$ is at least covered by $k$ work sensor node is

$$
P_{K}=1-\left(1-\frac{\pi\left(R_{0}^{2}+\lambda^{2}\right)}{\operatorname{area}(M)}\right)^{k}
$$

Assume the area covered by at least $k$ sensor nodes is $M^{\prime}$ and then the expectation of the area for $M^{\prime}$ is

$$
E\left(\operatorname{area}\left(M^{\prime}\right)\right)=P_{k} \operatorname{area}(M) .
$$

Therefore

$$
E\left(\eta_{k}\right)=\frac{E\left(\operatorname{area}\left(M^{\prime}\right)\right)}{\operatorname{area}\left(M^{\prime}\right)}=1-\left(1-\frac{\pi\left(R_{0}^{2}+\lambda^{2}\right)}{\operatorname{area}(M)}\right)^{k}
$$

With Theorem 5, we can get the expectation of the minimum number of required work sensor nodes satisfying demanded coverage quality $\eta_{d}$ of applications; namely,

$$
E(k)=\frac{\ln \left(1-\eta_{d}\right)}{\ln \left(1-\pi\left(R_{0}^{2}+\lambda^{2}\right) / \operatorname{area}(M)\right)} .
$$

Theorem 8. After the redundant sensor nodes are powered off randomly without causing any conflict, if the rest working nodes can provide the coverage quality, $n_{d}$ required by the application, the expectation for the number of redundant sensor nodes' neighbors satisfies

$$
\begin{aligned}
E(n)> & \frac{\ln \left(1-\eta_{d}\right)}{\ln \left(\left(1-\pi\left(R_{0}^{2}+\lambda^{2}\right) / \operatorname{area}(M)\right)-1\right)} \\
& \cdot \frac{4 \pi R_{0}^{2}+2 \pi \lambda^{2}}{\operatorname{area}(M)} .
\end{aligned}
$$

Proof. Assuming the number of the remaining work sensor nodes is $N$ after the redundant nods are powered off, then the $n$ work sensor nodes still follow random and uniform distribution. Because the remaining work sensor nodes can still accurately provide the coverage quality required in applications, by formula (22), we get

$$
E(N)=\frac{\ln \left(1-\eta_{d}\right)}{\ln \left(1-\pi\left(R_{0}^{2}+\lambda^{2}\right) / \operatorname{area}(M)\right)} .
$$

Denote the perception radius of sensor node as $R_{a}, R_{b}$, which represents the perception radius of sensor node $b$. If $b$ is the coverage neighbor of $a$, then $b$ must be located in a circle centered on the radius $R_{a}+R_{b}$ (denote the circle as $O\left(a, R_{a}+\right.$ $\left.R_{b}\right)$ ). Therefore, the probability that $b$ is a coverage neighbor of $a$ is

$$
P_{b-a}=\frac{\operatorname{area}\left(O\left(a, R_{a}+R_{b}\right)\right)}{\operatorname{area}(M)} .
$$

Because the perception radius of all sensor nodes obeys normal distribution $N\left(R_{0}, \lambda^{2}\right)$, as well as $R_{0} \geq 3.3 \lambda$, the probability that a sensor node is the coverage neighbor of another sensor node is

$$
\begin{aligned}
P & =\int_{0}^{2 R_{0}} \int_{0}^{2 R_{0}} P_{b-a} \\
& \cdot \frac{1}{2 \pi \lambda^{2}} \exp \left[-\frac{\left(R_{a}-R_{b}\right)^{2}+\left(R_{b}-R_{0}\right)^{2}}{2 \lambda^{2}}\right] \mathrm{d} R_{a} \mathrm{~d} R_{b} .
\end{aligned}
$$

Calculation is

$$
\begin{aligned}
P= & \frac{1}{\operatorname{area}(M)}\left[\int_{0}^{2 R_{0}} \int_{0}^{2 R_{0}} R_{a}^{2}\right. \\
& \cdot \frac{1}{\lambda^{2}} \exp \left[-\frac{\left(R_{a}-R_{b}\right)^{2}+\left(R_{b}-R_{0}\right)^{2}}{2 \lambda^{2}}\right] \mathrm{d} R_{a} \mathrm{~d} R_{b} \\
& +\int_{0}^{2 R_{0}} \int_{0}^{2 R_{0}} R_{a} R_{b} \\
& \left.\cdot \frac{1}{\lambda^{2}} \exp \left[-\frac{\left(R_{a}-R_{b}\right)^{2}+\left(R_{b}-R_{0}\right)^{2}}{2 \lambda^{2}}\right] \mathrm{d} R_{a} \mathrm{~d} R_{b}\right] .
\end{aligned}
$$
have

According to the computation process in formula (27), we

$$
\begin{aligned}
& \int_{0}^{2 R_{0}} \int_{0}^{2 R_{0}} R_{a}^{2} \\
& \cdot \frac{1}{\lambda^{2}} \exp \left[-\frac{\left(R_{a}-R_{b}\right)^{2}+\left(R_{b}-R_{0}\right)^{2}}{2 \lambda^{2}}\right] \mathrm{d} R_{a} \mathrm{~d} R_{b} \\
& \quad \approx \pi\left(R_{0}^{2}+\lambda^{2}\right) .
\end{aligned}
$$

Because

$$
\begin{aligned}
& \int_{0}^{2 R_{0}} R_{a} \frac{1}{(2 \pi)^{1 / 2} \lambda} \exp \left(-\frac{\left(R_{0}-R_{a}\right)^{2}}{2 \lambda^{2}}\right) \mathrm{d} R_{a} \\
& \quad=\int_{-R_{0} / \lambda}^{R_{0} / \lambda}\left(R_{0}+\lambda x\right) \frac{1}{(2 \pi)^{1 / 2}} \exp \left(-\frac{x}{2}\right) \mathrm{d} x=R_{0}
\end{aligned}
$$




$$
\begin{aligned}
& \int_{0}^{2 R_{0}} \int_{0}^{2 R_{0}} 2 \pi R_{a} R_{b} \\
& \cdot \frac{1}{2 \pi \lambda^{2}} \exp \left(-\frac{\left(R_{a}-R_{b}\right)^{2}+\left(R_{b}-R_{0}\right)^{2}}{2 \lambda^{2}}\right) \mathrm{d} R_{a} \mathrm{~d} R_{b} \\
& \quad=2 \pi R_{0}^{2} .
\end{aligned}
$$

Then we have

$$
P=\frac{4 \pi R_{0}^{2}+2 \pi \lambda^{2}}{\operatorname{area}(M)} .
$$

The expectation of the number of coverage neighbor sensor nodes for each of the remaining $N$ work sensor nodes is

$$
\begin{aligned}
E(n)= & (E(N)-1) P \\
= & \frac{\ln \left(1-\eta_{d}\right)}{\ln \left(\left(1-\pi\left(R_{0}^{2}+\lambda^{2}\right) / \operatorname{area}(M)\right)-1\right)} \\
& \cdot \frac{4 \pi R_{0}^{2}+2 \pi \lambda^{2}}{\operatorname{area}(M)} .
\end{aligned}
$$

Therefore the expectations of the number of redundant coverage neighboring sensor nodes satisfy

$$
\begin{aligned}
E(n)> & \frac{\ln \left(1-\eta_{d}\right)}{\ln \left(\left(1-\pi\left(R_{0}^{2}+\lambda^{2}\right) / \text { area }(M)\right)-1\right)} \\
& \frac{4 \pi R_{0}^{2}+2 \pi \lambda^{2}}{\operatorname{area}(M)} .
\end{aligned}
$$

3.3. Algorithm Description. As to the redundancy of sensor nodes, the connection graph of sensor nodes gathering redundancy is constructed, that is, the redundancy information calculated by a positioning algorithm (e.g., centroid positioning and ranging location) of all the nodes in the process of gathering and the neighbor nodes. The data collected in this cluster are sent to the sink node through communication links. After the sink nodes receive the redundancy information, it is calculated and analyzed. The connection graph $G$ of redundancy relation is constructed according to the results of calculation and analysis. The degree of connection graph $G$ is the number of limited sides related to vertex. It corresponds to the redundancy degree of sensor node. When the number of sensor nodes increases, the redundancy degree increases correspondingly. In the connection graph $G$, when the redundancy degree of sensor node surpasses the preset threshold value, the node transferred to the state of sleeping. At the same time, there exist several $(n>2)$ high redundancy rate nodes. If energy is taken as choosing condition, node with the most energy is chosen as the sleeping nodes by sort algorithm. And this node, together with the corresponding neighbor nodes, is cancelled in connection graph $G$. Iteration algorithm is used to iterate. Next node with most energy is found one
TABLE 1: The tabulation of the simulation parameters.

\begin{tabular}{lc}
\hline Parameter & Value \\
\hline Area I & $100 * 100$ \\
Area II & $200 * 200$ \\
Area III & $300 * 300$ \\
$R_{s}$ & $5 \mathrm{~m}$ \\
Energy & $5 \mathrm{~J}$ \\
Time & $800 \mathrm{~s}$ \\
$R_{c}$ & $10 \mathrm{~m}$ \\
$E_{R \text {-elec }}$ & $50 \mathrm{~J} / \mathrm{b}$ \\
$E_{T \text {-elec }}$ & $50 \mathrm{~J} / \mathrm{b}$ \\
$\varepsilon_{f s}$ & $10(\mathrm{~J} / \mathrm{b}) / \mathrm{m}^{2}$ \\
$\varepsilon_{\text {amp }}$ & $100(\mathrm{~J} / \mathrm{b}) / \mathrm{m}^{2}$ \\
$e_{\text {min }}$ & $0.005 \mathrm{~J}$ \\
\hline
\end{tabular}

by one until the redundancy degree of all sensor nodes is smaller than the threshold value in the connection graph G. As to energy consumption of sensor nodes, clustering algorithm is used to control the administrator nodes and member nodes, respectively. In the initial state of network, the member node transmits the datum of "inf_coverag" to gathering node. "inf_coverag" includes the information of location, ID, and energy. After one or several cycles, the gathering node receives the information of sensor nodes. The information is calculated. The node energy is stored in the chained list (CL) successively from the highest to the lowest one according to the rest node energy. The top nodes possess the covering ability of higher weight. Nodes are controlled according to the positioning information. The sensor node that satisfies the condition in the CL is sorted out and the message "inf_notice" is sent out to start the effective coverage. The rest nodes are in the state of sleeping to save the network energy expenses (see Algorithm 1).

\section{System Evaluations}

In order to verify the effectiveness and stability of the energyefficient coverage control with multinodes redundancy verification, ECMRV algorithm, this paper selects the MATLAB7 as the simulation platform. And the ECMRV algorithm is compared with the four algorithms of the literature [13] (the energy-efficient target coverage algorithm (ETCA)) and the literature [16] (energy-efficient $k$-coverage algorithm (EEKCA)), the literature [14] (event probability driven mechanism, EPDM), and the literature [17] (optimization strategy coverage control (OSCC)). And the simulation parameters are shown in Table 1.

Experiment 1. In terms of prolonging the network lifetime, the ECMRV algorithm is compared with the ETCA and the EEKCA, and the experimental data is the mean value of the 200 simulation data, as shown in Figures 3-5.

Experiment 1 is a contrast experiment of the ECMRV algorithm and the ETCA and the EEKCA in terms of the 


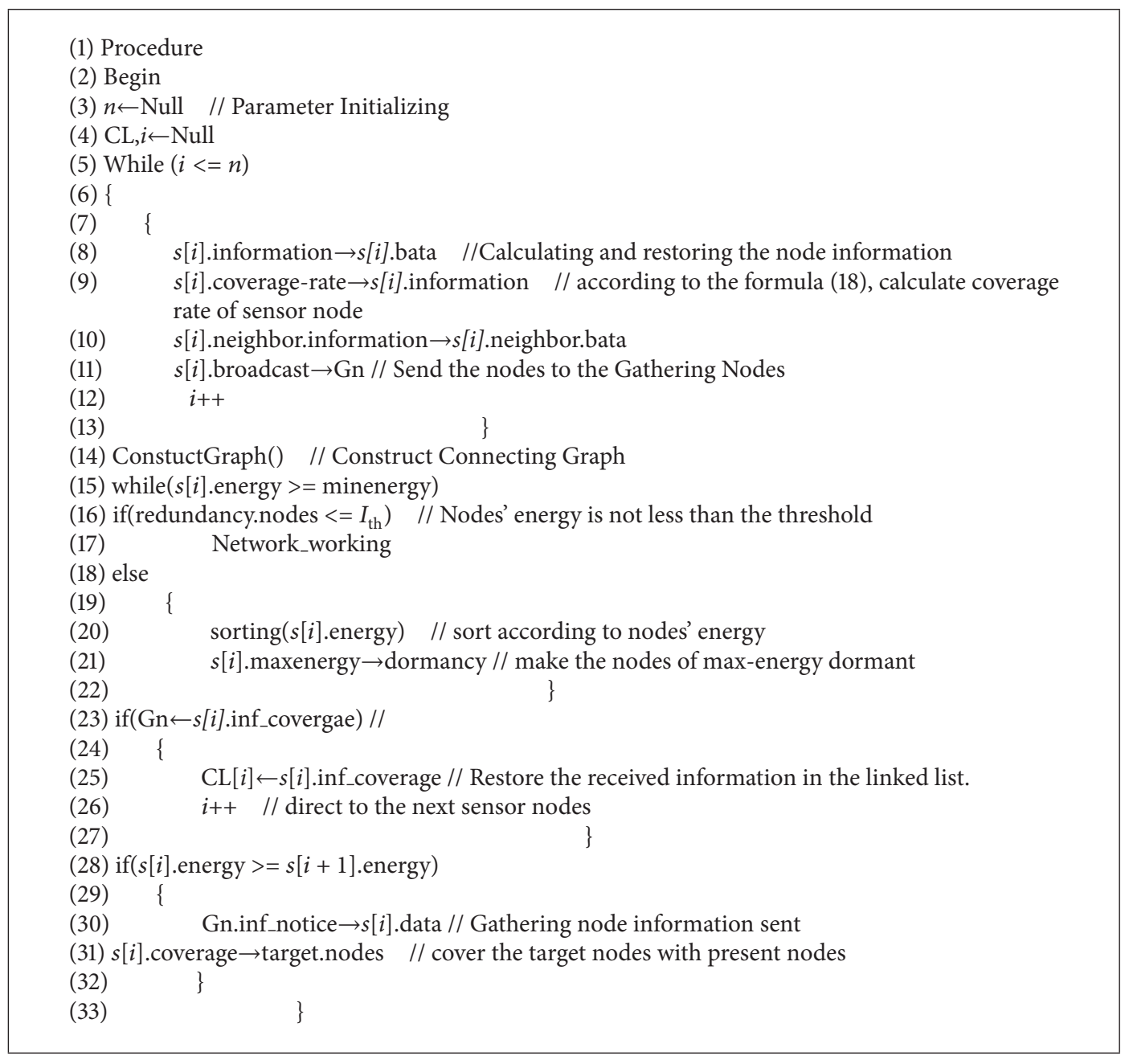

Algorithm 1: ECMRV algorithm description.

network lifetime in different monitoring areas. In the experiment, the values of $k$ are selected differently; the network scale can be changed by changing the number of the sensor nodes deployed randomly in the monitoring area. For the monitoring area of the smaller scale, the initial value of the number of the sensor nodes deployed randomly is 30 , based on which the number of the sensor nodes increases step by step. From the simulation, it can be seen that the lifetime of the Wireless Sensor Network is a linear rising trend with the increase of the number of the sensor nodes. The main reason is that the member sensor nodes in the sensor nodes set cover the target nodes by the scheduling mechanism of the sensor node. In the same network environment, compared with the ETCA and the EEKCA, the lifetime of the ECMRV algorithm has been prolonged by $11.29 \%$ on average; as for the larger scale of the monitoring area, the initial number of the sensor nodes is 50 , based on which the number of the sensor nodes increases step by step. The lifetime of the Wireless Sensor Network is also a linear rising trend with the increase of the number of the sensor nodes. The rise is more than that of the smaller monitoring area, and compared with the ETCA and the EEKCA, the network lifetime is increased by $16.12 \%$ and $15.96 \%$.

Experiment 2. The contrast experiment is taken between the ECMRV algorithm and the EPDM algorithm of the literature [14] and the OSCC algorithm of the literature [17] in terms of the coverage ratio and multitargets cycle control coverage preservation protocol [25] (MTCPP) in network running time. Taking the monitoring area of $200 * 200 \mathrm{~m}^{2}$ as example, the experimental data is the mean value of the 200 simulation data, as shown from Figures 6 to 8 .

In Figure 6, with the increase of the number of the sensor nodes, the coverage probabilities of the three algorithms are also increasing. When the coverage probability is $99.99 \%$ and $\lambda=2$, the number of the sensor node is 237; when $\lambda=3$, the number of the sensor nodes is 157; and when $\lambda=4$ and the number of the sensor nodes is 132 , the algorithm in this paper has reached 99.99\% coverage; however the EPDM algorithm and the OSCC algorithm have not reached $100 \%$ coverage, which shows that the coverage ratio of the ECMRV algorithm is higher than that of the EPDM algorithm and the OSCC 


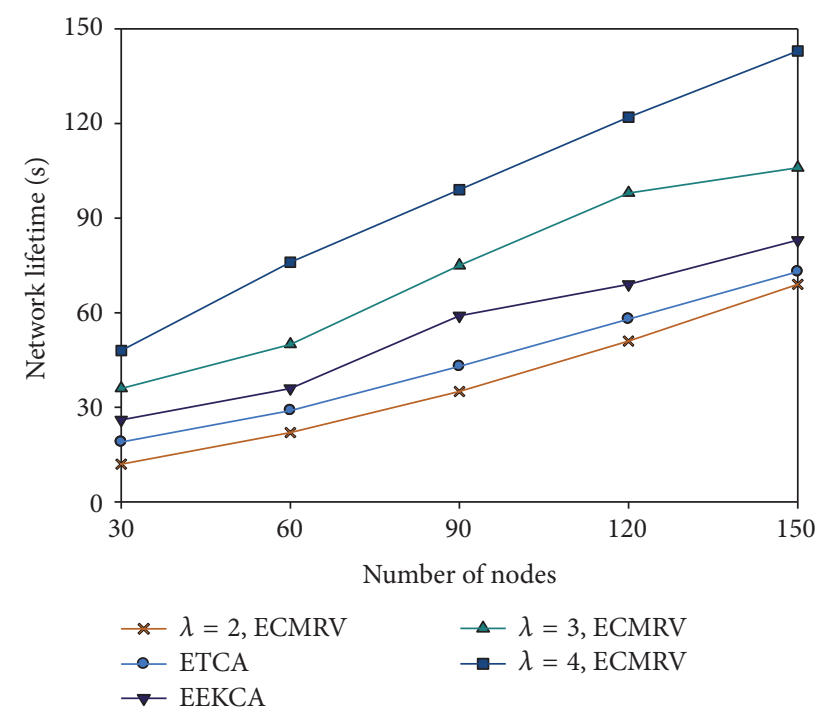

Figure 3: $100 * 100 \mathrm{~m}^{2}$, network lifetime.

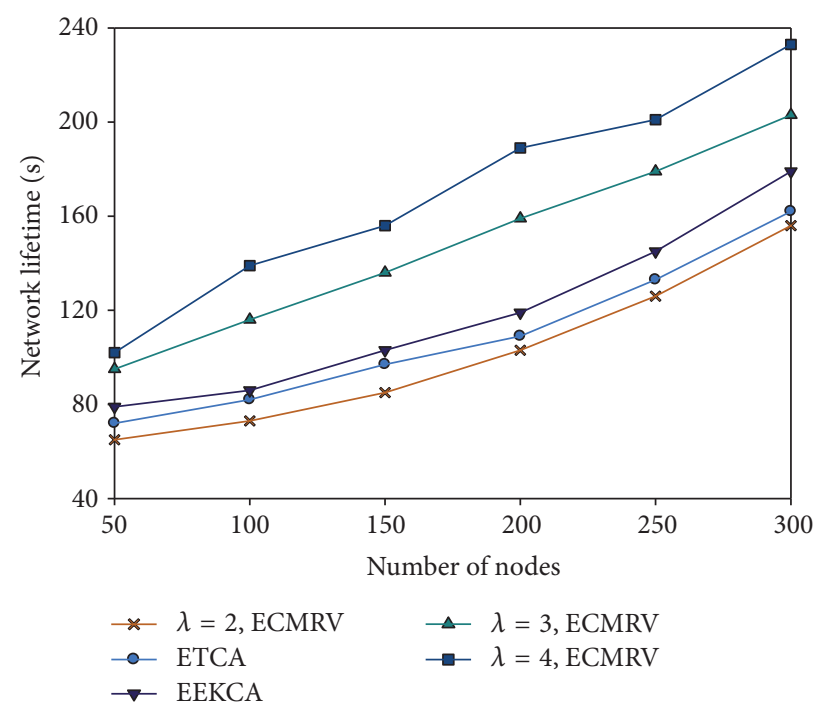

FIGURE 4: $200 * 200 \mathrm{~m}^{2}$, network lifetime.

algorithm. Thus, the effectiveness of the ECMRV algorithm in this paper has been verified. In Figure 7, at the initial time of the program execution, the coverage probabilities of the two algorithms are almost the same; however as time passes by, the coverage probabilities of the two contract algorithms are the declining trance. The main reason is that the EPDM algorithm and the OSCC algorithm adopt the uninterrupted coverage to complete the coverage during the run time; that is, the target nodes in the monitoring areas are covered constantly until the energies of the target nodes are consumed up. When $t=150$, the coverage probabilities of the three algorithms decline more obviously. When $\lambda=$ $2,3,4, \mathrm{CP}_{\mathrm{ECMRV} 2}=76.56 \%, \mathrm{CP}_{\mathrm{ECMRV} 3}=95.53 \%, \mathrm{CP}_{\mathrm{EPDM}}=$ $78.86 \%, \mathrm{CP}_{\mathrm{OSCC}}=84.02 \%$, and $\mathrm{CP}_{\mathrm{ECMRV}}=97.71 \%$. When $\lambda=4$, the coverage probability in this paper is higher than that of the EPDM algorithm and the OSCC algorithm,

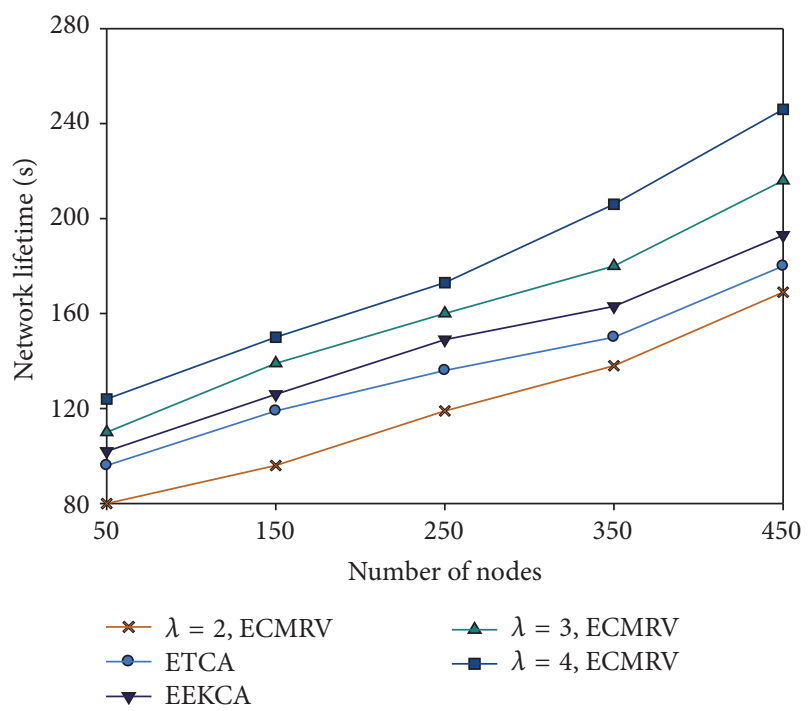

Figure 5: $300 * 300 \mathrm{~m}^{2}$, network lifetime.

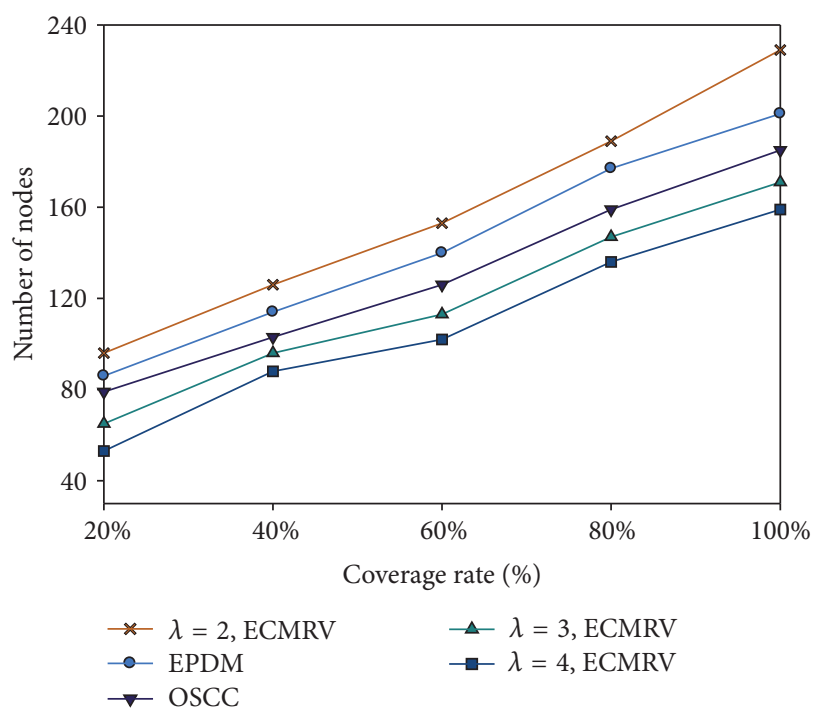

FIGURE 6: $200 * 200 \mathrm{~m}^{2}$, network coverage rate.

which shows that, with the function of the same sensor nodes, the coverage probability of the ECMRV algorithm is higher than that of the other two algorithms, which has verified the effectiveness of the algorithm in this paper. And Figure 8 gives the curves of the working sensor nodes and the sensor nodes contrast of the ECMRV algorithm and the EPDM algorithm and the OSCC algorithm, when the coverage probabilities are the same ones. When the number of sensor nodes is maintained from 260 to 290 , the numbers of sensor nodes of the three algorithms basically tend to be stable. When $\lambda=2,3,4$, the number of sensor nodes of the ECMRV algorithm is maintained at 245, 223, and 211, while the numbers of the sensor nodes in EPDM algorithm and the OSCC algorithm are maintained from 231 to 237. Therefore, the mean value of the number of sensor nodes of the ECMRV 


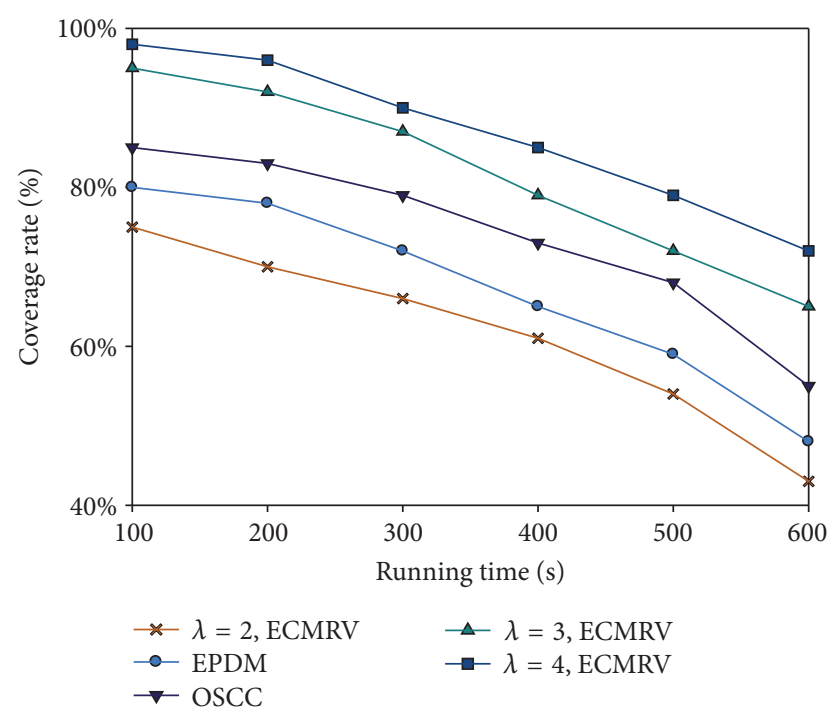

Figure 7: $200 * 200 \mathrm{~m}^{2}$, network running time.

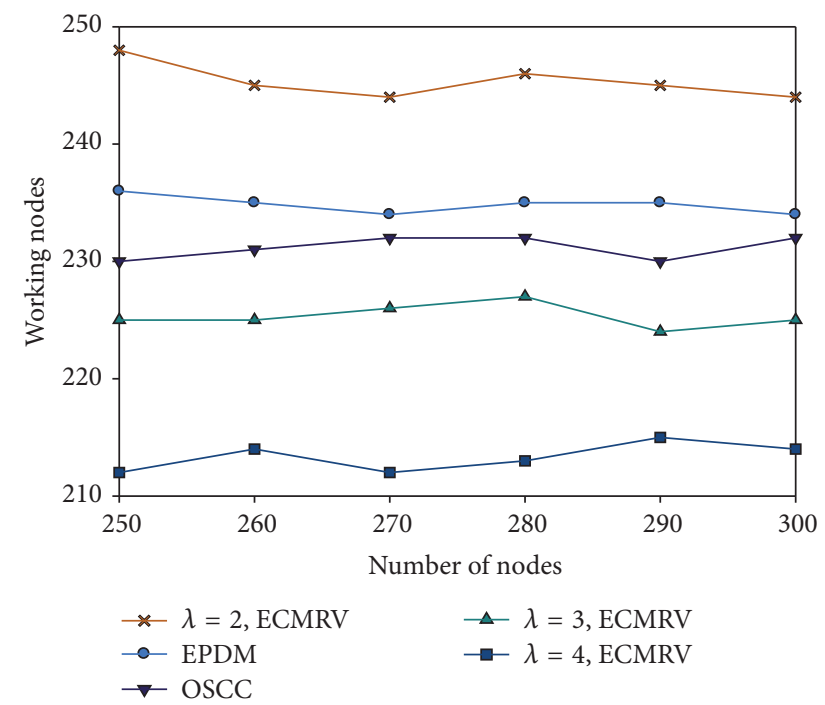

FIGURE 8: Number of sensor nodes and working sensor nodes.

algorithm is less than that of the other algorithms; the number of sensor nodes is reduced by $13.49 \%$.

Figure 9 provides the different network energy consumption in different monitoring area, and the contracted algorithms are the algorithms in the literature [13] and literature [25]. At the initial time of the three algorithms, the network lifetime is increasing with the increase of the sensor nodes. However, the parameter range limitation in this paper and the closing statement of the redundant sensor node make the network lifetime of the algorithm in this paper smaller than that of the other two algorithms, when the network energy reaches the balancing statement. Figure 10 provides the network lifetime and the target node. When the target nodes are being covered, the network energy of this paper is also smaller than that of the other two algorithms, and the reason is the same as the above situation. In Figure 11,

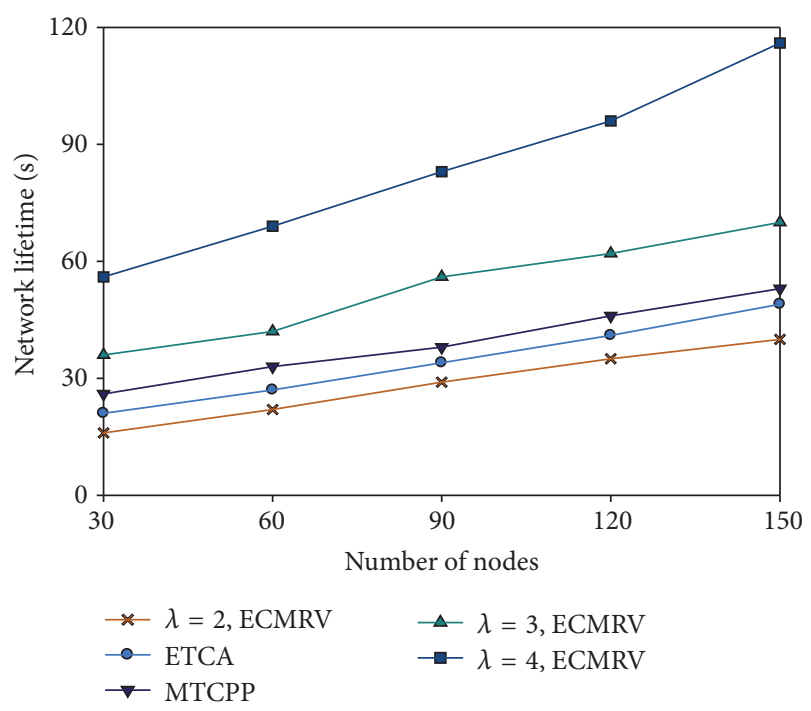

Figure 9: $100 * 100 \mathrm{~m}^{2}$, network lifetime and sensor nodes.

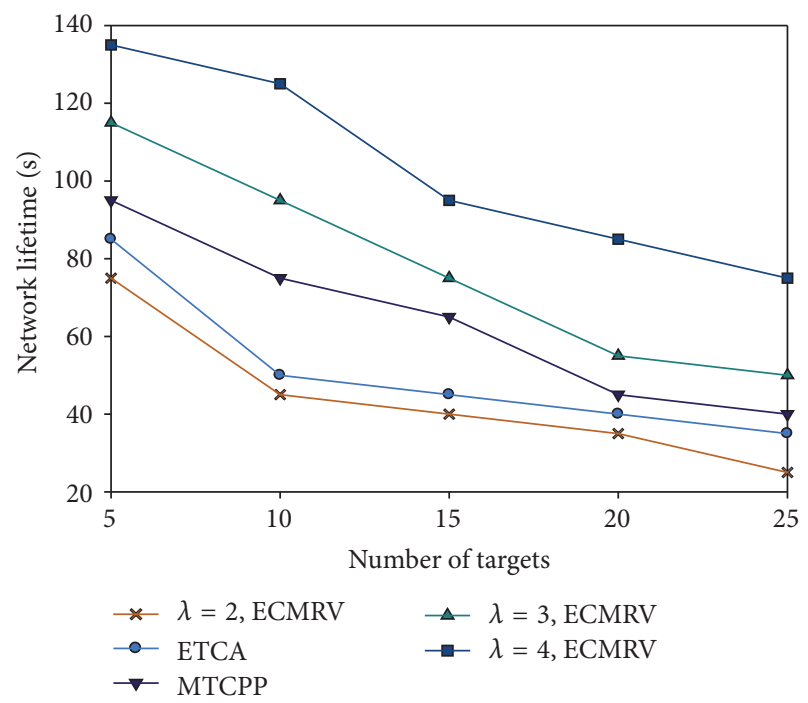

Figure 10: $100 * 100 \mathrm{~m}^{2}$, network lifetime and target nodes.

the expansion of the monitoring area makes some of the redundant sensor nodes in the working state, which has prolonged the network lifetime. When the parameter $\lambda=3$, the network lifetime of the algorithm in this paper is larger than that of the ETCA; when the parameter $\lambda=4$, the network lifetime is larger than that of the other algorithms; and Figure 12 provides the change of the network lifetime in the process of the coverage of the target node. With the increasing of the number of the target nodes, the network lifetime of the three algorithms has been the declining trance and finally provide the energy balance statement. But during the declining process, the average speed of the algorithm in this paper is smaller than that of the other algorithms, the main reasons are as follows. The coverage expectation of intensively deployed sensor nodes in the monitoring area is quite large. Meantime some redundant nodes are waken up to working nodes. Hence the coverage probability is enhanced 


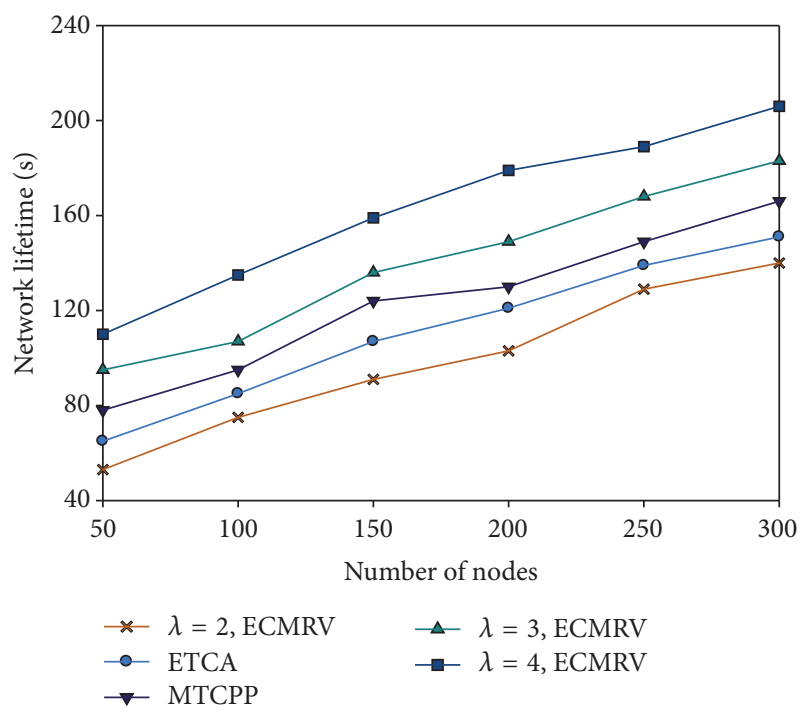

FIGURE 11: $200 * 200 \mathrm{~m}^{2}$, network lifetime and sensor nodes.

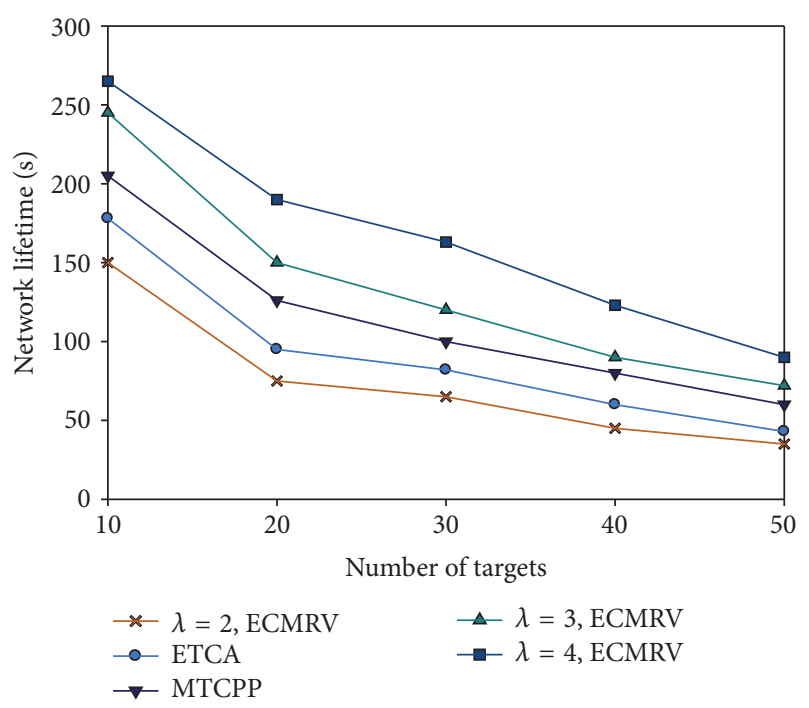

FIgURe $12: 200 * 200 \mathrm{~m}^{2}$, network lifetime and target nodes.

and the network lifetime is prolonged. It is also applicable for $300 * 300 \mathrm{~m}^{2}$. According to the analysis from Figures 812 , the algorithm in this paper has more energy during the running time; the reason is that, in the coverage process, the algorithm in this paper closes some of the redundant sensor nodes, which greatly prolongs the network lifetime. Thus, in the condition of the same sensor nodes numbers, the running time of the algorithm in this paper is a little higher than that of the other algorithms, as shown in Figures 13 and 14.

\section{Conclusion}

Based on the analysis of the coverage in the Wireless Sensor Network, this paper proposes an energy-efficient coverage algorithm with multinode redundancy verification (ECMRV). The algorithm provides the calculation method

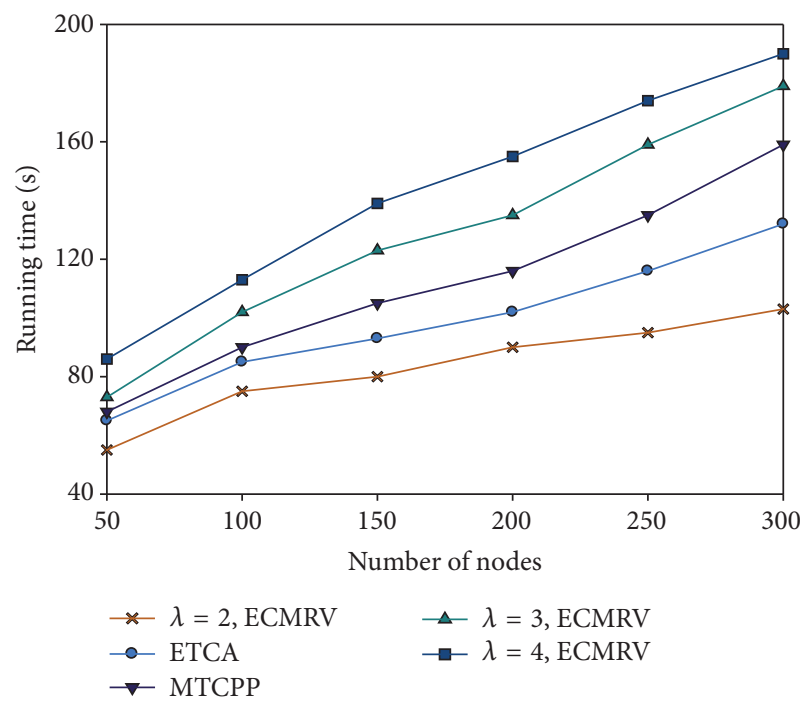

FIgURE 13: $200 * 200 \mathrm{~m}^{2}$, running time and sensor nodes.

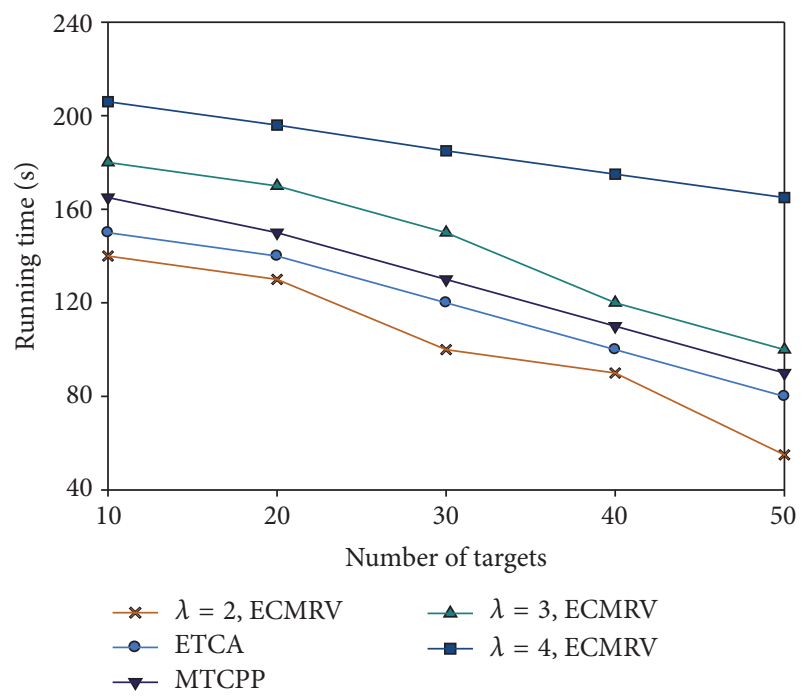

Figure 14: $200 * 200 \mathrm{~m}^{2}$, running time and target nodes.

with the expected value of the monitoring area, and, on this basis, it proves the solving process which uses the least sensor nodes to calculate the expected value of the monitoring area; meanwhile, the first coverage expected value of the moving target node is calculated and deduced. In terms of the energy, this paper has provided a detailed process to restrict the energy consumption, and, with the corresponding calculation, it has proved that the energy consumption of the multilateral transmission is less than that of the unilateral transmission. Finally, in the aspect of the different value ranges of $\lambda$, the network lifetime, and the network coverage, the simulation experiments are taken, and the results are compared with that of the ETCA, the EEKCA, the EPDM Algorithm, the OSCC algorithm, and MTCPP Algorithm, which finally verifies the effectiveness and the stability of the algorithm in this paper. 
The future work is to focus on how to realize the nonlinear programming for the effectiveness coverage of the multiple target nodes and how to improve the effective coverage of the boundary of the monitoring area.

\section{Competing Interests}

The authors declare no competing interests.

\section{Acknowledgments}

The study is supported by Projects U1304603 and 61503174 supported by the National Natural Science Foundation of China; Projects 15A413016, 16A520063, and 17A520044 supported by Henan Province Education Department Natural Science Foundation; Projects 152300410115, 152102410053, 162102210113,162102210276 , and 162102410051 supported by Natural Science and Technology Research of Foundation Project of Henan Province Department of Science; Project 1201430560 supported by Guangzhou Education Bureau Science Foundation; Project 2016A030313540 supported by Guangdong Natural Science Foundation of China. This paper is also supported by the National Scholarship Fund.

\section{References}

[1] J.-H. Seok, J.-Y. Lee, W. Kim, and J.-J. Lee, "A bipopulationbased evolutionary algorithm for solving full area coverage problems," IEEE Sensors Journal, vol. 13, no. 12, pp. 4796-4807, 2013.

[2] K. Derr and M. Manic, "Wireless sensor network configurationpart II: adaptive coverage for decentralized algorithms," IEEE Transactions on Industrial Informatics, vol. 9, no. 3, pp. 17281738, 2013.

[3] Z. B. Wang, J. L. Liao, Q. Cao, H. R. Qi, and Z. Wang, "Achieving $k$-barrier coverage in hybrid directional sensor networks," IEEE Transactions on Mobile Computing, vol. 13, no. 7, pp. 1443-1455, 2014.

[4] C. Yang and K.-W. Chin, "Novel algorithms for complete targets coverage in energy harvesting wireless sensor networks," IEEE Communications Letters, vol. 18, no. 1, pp. 118-121, 2014.

[5] M. R. Senouci, A. Mellouk, L. Oukhellou, and A. Aissani, "An evidence-based sensor coverage model," IEEE Communications Letters, vol. 16, no. 9, pp. 1462-1465, 2012.

[6] Y. Li, C. Vu, C. Ai, G. Chen, and Y. Zhao, "Transforming complete coverage algorithms to partial coverage algorithms for wireless sensor networks," IEEE Transactions on Parallel and Distributed Systems, vol. 22, no. 4, pp. 695-703, 2011.

[7] F.-Z. Meng, H.-Z. Wang, and H. He, "Connected coverage protocol using cooperative sensing model for wireless sensor networks," Acta Electronica Sinica, vol. 39, no. 4, pp. 772-779, 2011.

[8] S. Mini, S. K. Udgata, and S. L. Sabat, "Sensor deployment and scheduling for target coverage problem in wireless sensor networks," IEEE Sensors Journal, vol. 14, no. 3, pp. 636-644, 2014.

[9] D. W. Gong and Y. Y. Yang, "Low-latency SINR-based data gathering in wireless sensor networks," IEEE Transactions on Wireless Communications, vol. 13, no. 6, pp. 3207-3221, 2014.
[10] H. Mahboubi, K. Moezzi, A. G. Aghdam, K. Sayrafian-Pour, and V. Marbukh, "Distributed deployment algorithms for improved coverage in a network of wireless mobile sensors," IEEE Transactions on Industrial Informatics, vol. 10, no. 1, pp. 163-175, 2014.

[11] Y.-C. Tseng, P.-Y. Chen, and W.-T. Chen, "k-angle object coverage problem in a wireless sensor network," IEEE Sensors Journal, vol. 12, no. 12, pp. 3408-3416, 2012.

[12] Z. Wang, J. Liao, Q. Cao, H. Qi, and Z. Weng, "Achieving kbarrier coverage in hybrid directional sensor networks," IEEE Transactions on Mobile Computing, vol. 13, no. 7, pp. 1443-1455, 2014.

[13] X. Xing, G. Wang, and J. Li, "Polytype target coverage scheme for heterogeneous wireless sensor networks using linear programming," Wireless Communications and Mobile Computing, vol. 14, no. 14, pp. 1397-1408, 2014.

[14] Z. Y. Sun, W. G. Wu, H. Z. Wang, H. Chen, and X. F. Xing, "A novel coverage algorithm based on eventprobability-driven mechanism in wireless sensor network," Eurasip Journal on Wireless Communications and Networking, vol. 2014, article 58, 2014.

[15] C.-H. Wu, K.-C. Lee, and Y.-C. Chung, "A Delaunay Triangulation based method for wireless sensor network deployment," Computer Communications, vol. 30, no. 14-15, pp. 2744-2752, 2007.

[16] C. Li, Z. Sun, H. Wang, and H. Song, "A novel energy-efficient $k$ Coverage algorithm based on probability driven mechanism of wireless sensor networks," International Journal of Distributed Sensor Networks, vol. 2016, Article ID 7474926, 9 pages, 2016.

[17] Z. Y. Sun, W. G. Wu, H. Z. Wang, H. Chen, and W. Wei, "An optimized strategy coverage control algorithm for WSN," International Journal of Distributed Sensor Networks, vol. 2014, Article ID 976307, 12 pages, 2014.

[18] C. J. Zhao, H. R. Wu, Q. Liu, and L. Zhu, "Optimization strategy on coverage control in wireless sensor network based on Voronoi," Journal of Communications, vol. 28, no. 1, pp. 3643, 2013.

[19] Z. Y. Sun, X. F. Xing, C. F. Li, Y. L. Nie, and Y. J. Cao, "A novel nonlinear multitarget $\kappa$-degree coverage preservation protocol in wireless sensor networks," Journal of Sensors, vol. 2016, Article ID 3434961, 13 pages, 2016.

[20] Z. Lu, W. W. Li, and M. Pan, "Maximum lifetime scheduling for target coverage and data collection in wireless sensor networks," IEEE Transactions on Vehicular Technology, vol. 64, no. 2, pp. 714-727, 2015.

[21] C.-T. Cheng, H. Leung, and P. Maupin, "A delay-aware network structure for wireless sensor networks with in-network data fusion," IEEE Sensors Journal, vol. 13, no. 5, pp. 1622-1631, 2013.

[22] S. Zairi, B. Zouari, E. Niel, and E. Dumitrescu, "Nodes selfscheduling approach for maximising wireless sensor network lifetime based on remaining energy," IET Wireless Sensor Systems, vol. 2, no. 1, pp. 52-62, 2012.

[23] C. Menegazzo and L. C. P. Albini, "Unadvertised energy saving method for static and homogeneous wireless sensor networks," IET Wireless Sensor Systems, vol. 4, no. 3, pp. 105-111, 2014.

[24] L. H. Kong, M. C. Zhao, X.-Y. Liu et al., "Surface coverage in sensor networks," IEEE Transactions on Parallel and Distributed Systems, vol. 25, no. 1, pp. 234-243, 2014.

[25] Y. P. Li and Y. L. Yun, "A novel multi-targets cycle control coverage preservation protocol in wireless sensor network," International Journal of Future Generation Communication and Networking, vol. 9, no. 6, pp. 17-28, 2016. 


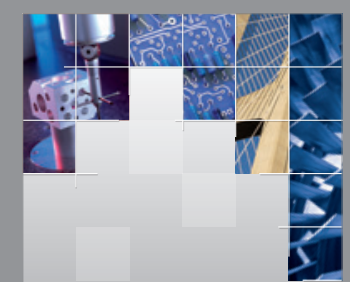

\section{Enfincering}
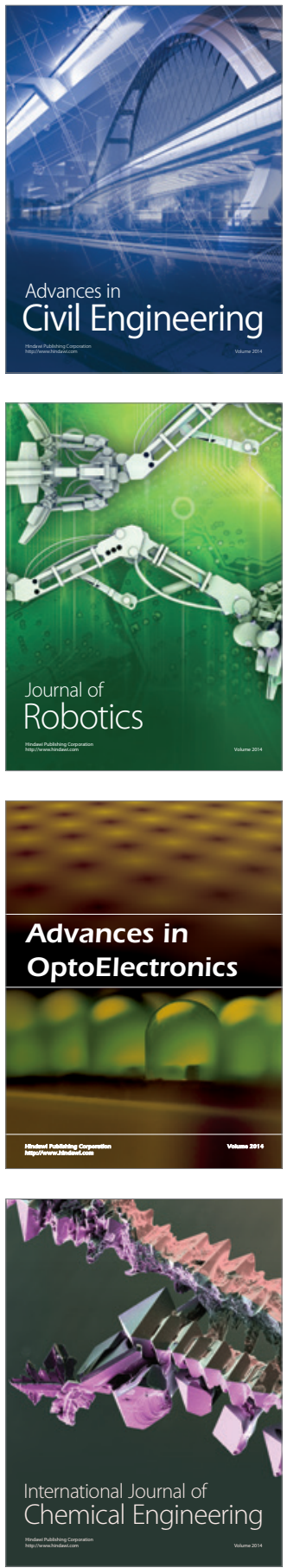

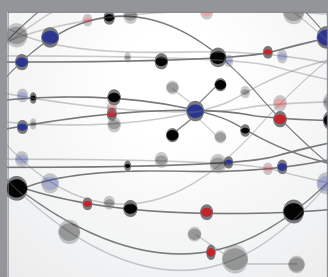

The Scientific World Journal

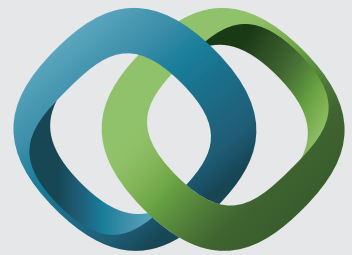

\section{Hindawi}

Submit your manuscripts at

http://www.hindawi.com
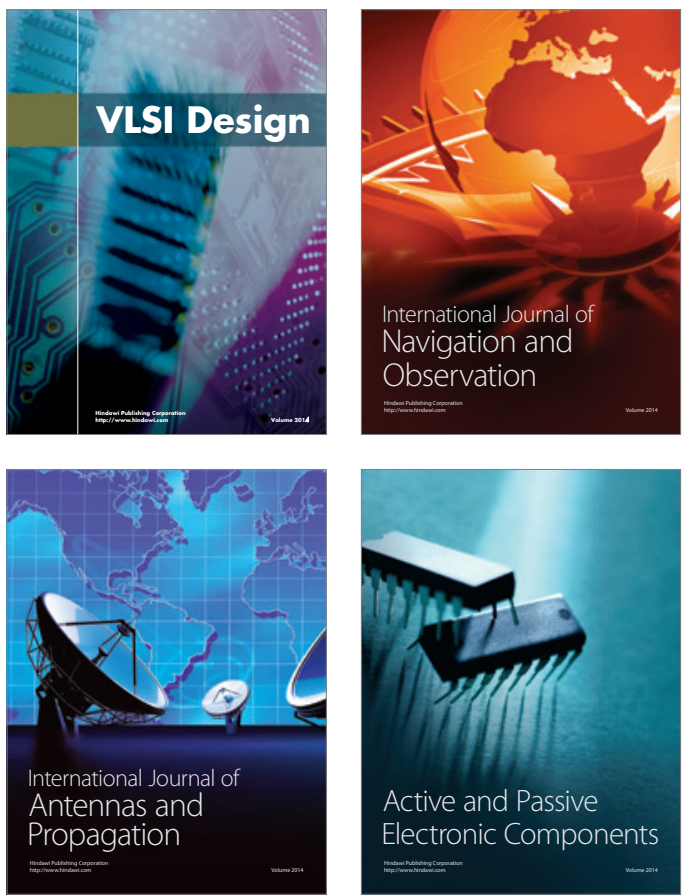
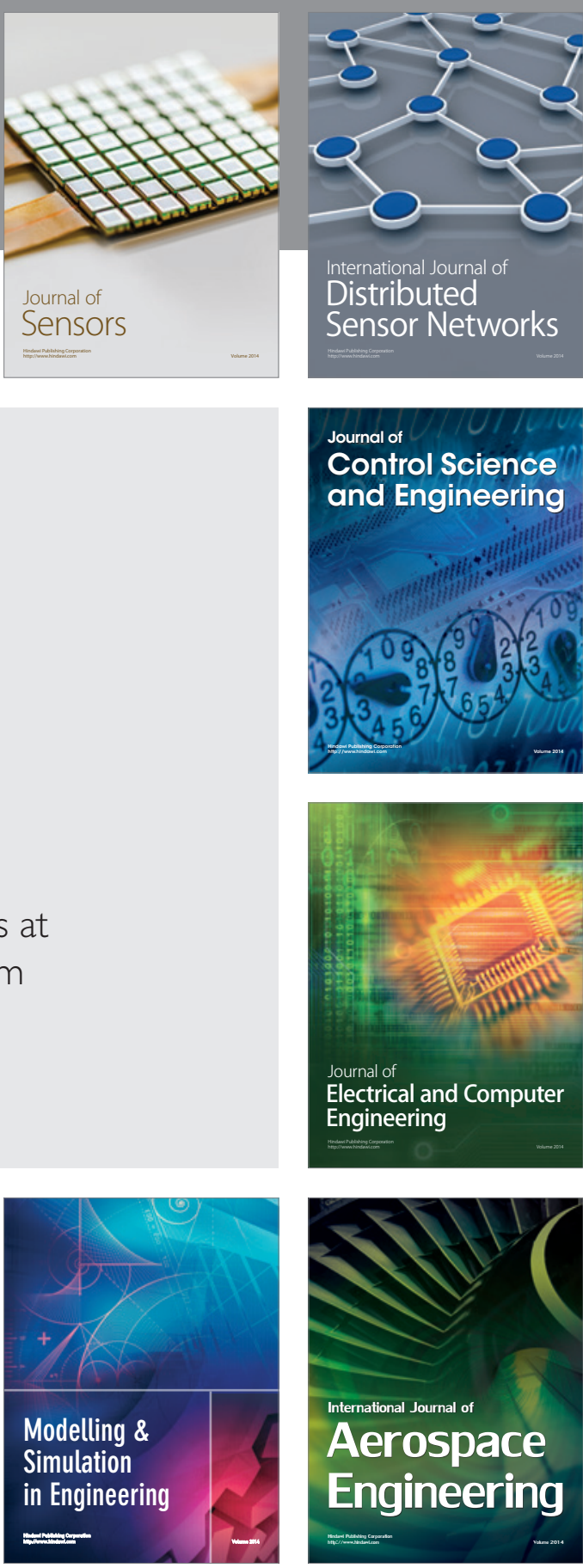

International Journal of

Distributed

Sensor Networks

Journal of

Control Science

and Engineering
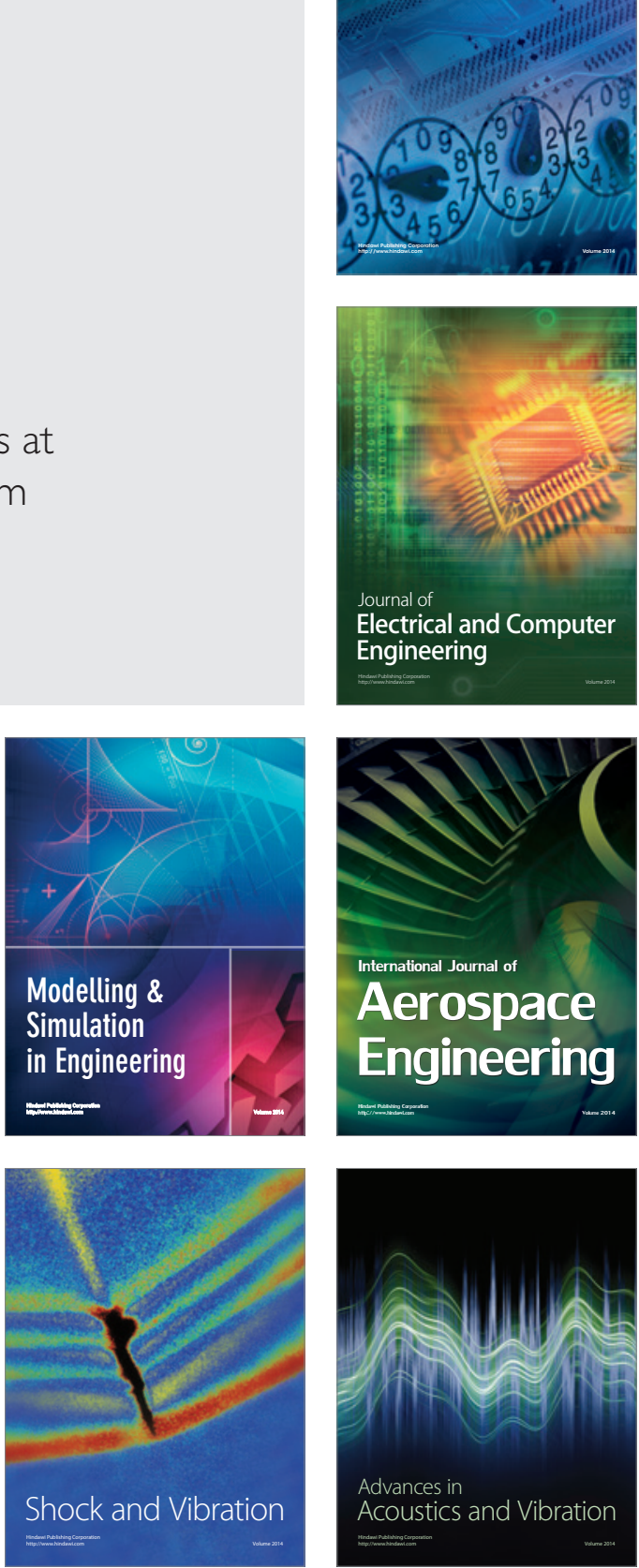